\title{
Antonio Gómez Marcano. La tesis doctoral sobre la encefalitis guanidínica (1933/1934) y notas sobre su exilio venezolano
}

\author{
Francisco Herrera Rodríguez \\ Catedrático de Escuela Universitaria jubilado (Historia de la Enfermería y \\ Fundamentos e Historia de la Fisioterapia). Facultad de Enfermería y \\ Fisioterapia (Universidad de Cádiz) \\ francisco.herrera@uca.es
}

RESUMEN: Los objetivos de este trabajo son dos: presentar una síntesis del expediente académico de Antonio Gómez Marcano en la Facultad de Medicina de Cádiz y estudiar los principales puntos de su tesis doctoral sobre la encefalitis guanidínica, defendida en Madrid en 1933, y publicada en la revista Archivos de Neurobiología, en 1934, cuyo Consejo de Redacción estaba presidido por Santiago Ramón y Cajal. Asimismo realizamos una síntesis sobre la labor de Gómez Marcano en la lucha contra la malaria durante su exilio en Venezuela.

Palabras clave: Antonio Gómez Marcano, Histopatología, Encefalitis Guanidínica, Médicos exiliados.

\section{Antonio Gómez Marcano. The doctoral thesis on guanidinic encephalitis (1933/1934) and notes on his Venezuelan exile}

ABSTRACT: The objectives of this work are two: to present a synthesis of the academic record of Antonio Gómez Marcano at Faculty of Medicine in Cádiz and to study the main points of his doctoral thesis on guanidinic encephalitis, defended in Madrid in 1933, and published in the journal Archives of Neurobiology, in 1934, whose Editorial Board was chaired by Santiago Ramón y Cajal. We have also made a synthesis on the work of Gómez Marcano about the fight against malaria during his exile period in Venezuela.

DOI del artículo:
http://dx.doi.org/10.25267/Cuad investig fondos arch UCA.2019.i1
$\underline{.03}$

DOI del artículo: .03

\section{Editorial ad UCA}


Keywords: Antonio Gómez Marcano, Histopahology, Guanidinic Encephalitis, Exiled doctors.

\footnotetext{
“A la voluntad, más que a la inteligencia, se enderezan nuestros consejos, porque tenemos la convicción de que aquélla, como afirma Payot, es tan educable como ésta, y creemos además que toda obra grande, en arte como en ciencia, es el resultado de una gran pasión puesta al servicio de una gran idea”.
}

Santiago Ramón y Cajal, Los tónicos de la voluntad.

“En su clásico tratado 'La estructura de las revoluciones cientificas', Thomas S. Khun sostiene que la ciencia avanza gracias a dos factores diametralmente opuestos: el "revolucionario” y el que él llama de "ciencia normal".

Rita Levi-Montalcini, Elogio de la imperfección.

A la Memoria del Dr. D. Manuel Gutiérrez Rodríguez

Este artículo se sitúa en la línea de investigación que venimos desarrollando desde la década de los ochenta, cuyo objetivo fundamental es estudiar la evolución del doctorado en la Facultad de Medicina de Cádiz, además de conocer las ideas médicas expresadas en las tesis doctorales de médicos gaditanos, españoles e hispanoamericanos. Nuestra intención, pues, ha sido y es comprender cómo ha evolucionado el grado de doctor a través de los planes de estudio de Medicina y rescatar de diversos archivos tesis doctorales que normalmente han sido poco valoradas en el contexto de la investigación científico médica en España. En estos trabajos hemos dado noticias de tesis doctorales librescas, en las que se hacen revisiones del tema en cuestión, aunque algunos autores también ofrecían su opinión personal, todo ello porque la legislación vigente no exigía otra cosa; pero también hemos localizado tesis, elaboradas a partir de finales del siglo XIX, que se realizaron desde una perspectiva clínica o experimental, en consonancia con los planes de estudio que iban apareciendo y que marcaban pautas nuevas para el doctorado, de todos estos trabajos dejamos constancia en la bibliografía por si fueran de utilidad a algún investigador, incluyendo el estudio de una tesis doctoral argentina sobre la epidemia de gripe de 1918-1919 (Herrera Rodríguez, 1987a; 1987b; 198889; 1991; 1992; 1995; 2000; 2006; 2009a; 2014) (Herrera Rodríguez y Cabrera Afonso 1986) (Herrera Rodríguez, Cabrera Afonso y Márquez Espinós 1988) (Márquez Espinós y Herrera Rodríguez 1989). No podemos olvidar aquí el empuje y aliento recibidos en esta línea de investigación por parte de nuestro maestro, el profesor Antonio Orozco Acuaviva (19342000), y la labor que realizó personalmente compilando libros, monografías y tesis en su Bibliografía Médico-Científica gaditana (Orozco 1981). Una vez apuntadas estas precisiones introductorias centramos nuestros comentarios en el tema que nos ocupa en este artículo.

DOI del artículo:
http://dx.doi.org/10.25267/Cuad investig fondos arch UCA.2019.i1
.$\underline{.03}$

DOI del artículo: .03 
La tesis doctoral sobre la encefalitis guanidínica de Antonio Gómez Marcano (19051983), elaborada durante la II República, en Cádiz, y defendida en Madrid como obligaba la legislación vigente, es un trabajo de carácter experimental, realizado como comentaremos más adelante bajo el magisterio de Luis Urtubey Rebollo (1892-1962) (Gómez Sánchez 1980, p. 169; Gómez Sánchez 2008; Herrera Rodríguez 2011, p. 449-459), y por tanto teniendo muy presente el legado de Santiago Ramón y Cajal (1852-1934) y de Pío del Río Hortega (18821945) (Cannon 1981; López Piñero 1985; Laín Entralgo 2008; Riera Palmero, 2005; Riera Palmero y Del Río Hortega 2004). Para una mayor claridad expositiva este artículo lo vertebramos en tres apartados: en el primero damos noticias del expediente académico de Antonio Gómez Marcano en la Facultad de Medicina de Cádiz, que hemos podido consultar en el Archivo de la Universidad de Cádiz; en el segundo, nos centramos en comentar los puntos más notables de su tesis doctoral, de la cual poseemos en nuestro Archivo particular un ejemplar de la revista Archivos de Neurobiología del año 1934 en la que se incluye, así como la edición de la misma en formato de separata, de todo ello damos noticia puntual en Fuentes y Bibliografía. Y, por último, un tercer apartado en que ofrecemos una visión general sobre la labor de Marcano en los años 30, la Guerra Civil española y su exilio en Venezuela.

\section{LA CARRERA EN la FACUltad DE MEDicina DE CÁDIZ}

El profesor Francisco Guerra, cuando estaba elaborando su libro "La medicina en el exilio republicano", en el que incluyó una breve síntesis biográfica de Antonio Gómez Marcano a la que haremos referencia más adelante, nos hizo el encargo de buscar las fechas de su nacimiento y muerte, esta circunstancia nos permitió comprobar que nació en San Fernando (Cádiz), el 27 de mayo de 1905, y que falleció en Cádiz el 5 de julio de 1983. En un certificado de 26 de agosto de 1923, del secretario del Ayuntamiento de Cádiz, que se puede consultar en el Archivo de la Universidad de Cádiz, consta también el lugar y fecha de nacimiento citados, así como que era hijo de don Enrique Gómez y de doña Victoria Marcano, además de ofrecer alguna información complementaria sobre su incorporación al servicio militar. Dice así: “...en razón de su edad y con arreglo a lo prevenido en la vigente ley de Reclutamiento y reemplazo del Ejército no debe ser comprendido en alistamiento de quintas hasta el año de mil novecientos veintiséis".

En su expediente de alumno de Medicina, encontramos también un documento mecanografiado en el que se precisan las asignaturas y las calificaciones que obtuvo a lo largo de la carrera de Medicina, asimismo consta que en el curso 1920-21 cursó en la Escuela Profesional de Comercio de Cádiz la asignatura "Alemán $1^{\circ}$ curso" con la calificación de Sobresaliente. Sabemos también por esta documentación que vivía en Cádiz en la calle Rosario 10 dpdo., y que en 1919 estaba “vacunado y revacunado con resultado positivo". Este hecho era muy importante para cualquier ciudadano, estar vacunado contra la viruela, pero es evidente que un sanitario (médico, enfermera, practicante, etc.), o un alumno de medicina, tenían un riesgo añadido al tratar con estos pacientes en los pabellones de

DOI del artículo:
http://dx.doi.org/10.25267/Cuad investig fondos arch UCA.2019.i1
$\underline{.03}$
Editorial ad UCA 
enfermedades infecciosas. Del Archivo de la Universidad de Cádiz reproducimos el siguiente documento en el cual Antonio Gómez Marcano solicita el 30 de septiembre de 1921, se le tenga en cuenta en el pago el haber obtenido 2 Matrículas de Honor, aspecto en el que insistiremos un poco más adelante en este artículo (Imagen 1).

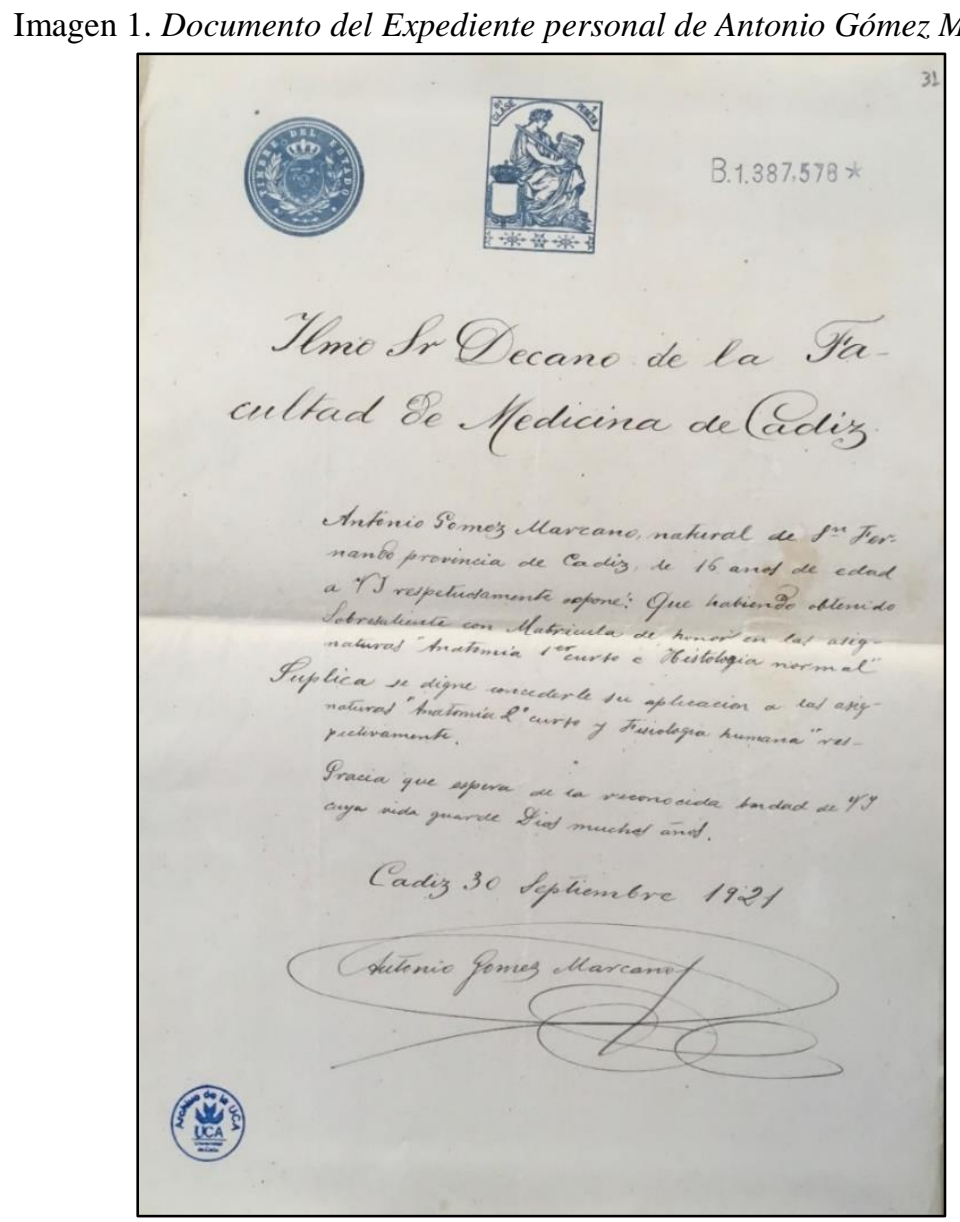

Fuente: Archivo de la Universidad de Cádiz (AUCA), C-211(3)-4 FM.

En su expediente personal de alumno de doctorado en Madrid, que puede consultarse en el Archivo Histórico de la Universidad Complutense (Expedientes académicos. Medicina, Ca 286), hemos localizado un certificado académico detallado y completo, firmado en Cádiz, el 25 de marzo de 1933, con el visto bueno del Decano de la Facultad de Medicina de esta ciudad, Leonardo Rodrigo Lavín (1867-1950). En este documento consta que realizó los "Estudios preparatorios", en el curso 1919-1920, cursando las asignaturas Física General, Química General, Biología y Geología. Continuó los estudios en el curso 1920-21 y concluyó la carrera en el curso 1924-25 cuando contaba 20 años de edad. En este certificado podemos apreciar la brillante carrera que realizó Antonio Gómez Marcano en Cádiz, tanto en los estudios preparatorios como en la licenciatura. Contabilizamos dos "Aprobado", en las asignaturas de Física General y Obstetricia-Ginecología con su clínica-2º dos "Notable", en Anatomía descriptiva-Embriología $2^{\circ}$ y Técnica Anatómica $2^{\circ}$; y un total de veintiséis

DOI del artículo:
$\underline{\text { http://dx.doi.org/10.25267/Cuad investig fondos arch UCA.2019.i1 }}$
$\underline{.03}$


"Sobresaliente", de los cuales veintiuno lo son con "Matrícula de Honor". Entre estas asignaturas en las que obtiene la máxima calificación podemos citar, por ejemplo, Histología e Histoquimia normales, Fisiología humana teórica y experimental, Anatomía patológica, Higiene con prácticas de Bacteriología sanitaria, etc. Este mismo certificado nos informa que en junio de 1925 efectuó los ejercicios de Reválida de la licenciatura obteniendo la calificación de Sobresaliente. Su expediente de alumno de Medicina, consultado en el Archivo de la Universidad de Cádiz, nos ofrece además una información complementaria, ya que en su interior se encuentran los dos ejercicios manuscritos de los temas que desarrolló en este examen de reválida de la licenciatura, efectuados como decimos en junio de 1925. Los temas a los que aludimos son los siguientes: "Papel del suelo en las epidemias" y "Tétanos traumático".

Hemos podido comprobar que un año después de concluir la carrera, en 1926, el Diario Oficial del Ministerio de Marina del día 30 de abril publicó una Real Orden firmada por Cornejo, Inspector Jefe de la Sección de Sanidad, en la que se informa que han aprobado 14 candidatos las oposiciones de ingreso al Cuerpo de Sanidad de la Armada, figurando el primero Antonio Gómez Marcano; también se ofrece esta noticia con la relación de los opositores aprobados en el periódico El Eco de Cartagena del día 1 de mayo del referido año; tiene en ese momento el joven médico de San Fernando tan solo 21 años de edad, y como podemos apreciar después de una licenciatura brillantísima en la Facultad de Medicina de Cádiz, demuestra su capacidad e inteligencia en estas oposiciones que le dan acceso al Cuerpo de Sanidad de la Armada.

Tiene, pues, a muy temprana edad, la licenciatura y también estabilidad profesional en la Armada, pero Gómez Marcano aspira también al grado de doctor en Medicina, y en estos años que estamos glosando había que realizarlo en la Universidad de Madrid, afortunadamente su expediente académico en esta Universidad, referenciado en el apartado de Fuentes, nos informa que en el curso 1930-31 se matriculó y examinó de las asignaturas del doctorado que en estas fechas eran las siguientes: Historia de la Medicina (Aprobado); Hidrología (Sobresaliente); Análisis Químico (Aprobado) y Parasitología (Sobresaliente).

\section{LA TESIS DOCTORAL SOBRE LA ENCEFALITIS GUANIDÍNICA (1933/34)}

Una vez aprobadas en 1931 las asignaturas del doctorado, Antonio Gómez Marcano ya está en situación de defender su tesis doctoral; pero no será hasta el día 22 de febrero de 1933 cuando curse una instancia al Decano de la Facultad de Medicina de Madrid solicitando se le admita a los ejercicios del grado de doctor, ya que tiene aprobadas las asignaturas de doctorado y ha abonado "la cantidad de treinta y siete pesetas con cincuenta céntimos en papel de pagos al estado por los derechos de inscripción”. El Decano accidental, en esos momentos el profesor Eduardo García del Real (1870-1947) (Díaz Rubio 2003, p. 70-71), autoriza el 8 de marzo de 1933 que se fije día, hora y local para verificar la defensa de la tesis doctoral. Finalmente, según consta en el acta del grado de doctor, el día 8 de mayo de 1933, el

DOI del artículo:
http://dx.doi.org/10.25267/Cuad investig fondos arch UCA.2019.i1
$\underline{.03}$

DOI del artículo: .03 
aspirante "leyó su Memoria doctoral" sobre "Encefalitis guanidínica", obteniendo la calificación de "Sobresaliente". Entre los miembros del tribunal que juzgó la tesis encontramos como vocal a la profesora Antonia Martínez Casado, que en esos años impartía en la Facultad de Medicina la docencia de Hidrología Médica, asignatura del doctorado, autora junto a Arturo Cervignon de un Manual de prácticas hidrológicas publicado en Madrid en 1931 (Rodríguez Sánchez et al. 2012, p. 280 y 286; Rodríguez Sánchez 1998, p. 270). En su expediente de doctorado consta además que, en Madrid, el 20 de mayo de 1954, "habiendo sido abonada la cantidad de mil ochenta pesetas en papel de pagos al Estado, el Ilmo. Sr. Decano solicita con esta fecha del Ilmo. Sr. Rector se sirva elevar a la Superioridad el expediente para la expedición del correspondiente Título de Doctor en la Facultad de Medicina". Un título que con "el número $102 f^{0} 168$ del Registro de la Subsecretaría", fue remitido a Cádiz el 1 de junio de 1955. Nada menos que 22 años transcurren entre la fecha del examen de grado de doctor, 1933, y la fecha de recepción del título en 1955. Todo ello debe atribuirse en parte al exilio de Antonio Gómez Marcano, primero en Orán y posteriormente en Venezuela, después de los trágicos sucesos de la Guerra civil (Guerra 2003, p. 728). Cabe pensar, como circunstancia más lógica, que algún familiar o amigo abonara las tasas y realizara los trámites burocráticos para la expedición del título de doctor y que se lo enviara a Venezuela en 1955, sobre este aspecto concreto del exilio nos ocuparemos en el apartado final de este artículo.

Una vez aclarado estos pormenores sobre la realización de los estudios del doctorado y la defensa de la tesis doctoral en Madrid, en 1933, conviene que señalemos antes de comentar los principales aspectos de la tesis, en qué grupo de trabajo se incardinó Antonio Gómez Marcano para llevar a buen término la misma, así como cuál era su actividad profesional en esos años en que la estaba gestando. En este sentido podemos indicar que una vez que termina la carrera en 1925, como hemos indicado, ingresó al año siguiente en la Armada como Teniente Médico, "estuvo embarcado en el crucero « Reina Regente» y llegó a capitán médico en 1928”, (Guerra 2003b, p. 728). En el encabezamiento de su tesis doctoral, Antonio Gómez Marcano, señala efectivamente que es Capitán Médico de la Armada y "Asistente del Laboratorio de Histología normal y patológica de la Facultad de Medicina de Cádiz”. De hecho el profesor Guerra apunta que en 1933 es Auxiliar de Anatomía Patológica en Cádiz con el profesor Luis Urtubey, y "en 1934 profesor auxiliar y pensionado dos años en Alemania por la Junta de Ampliación de Estudios” (2003, p. 728).

Efectivamente, Gómez Sánchez ha señalado a Antonio Gómez Marcano como discípulo directo de Luis Urtubey (catedrático de Histología, primero en Cádiz y posteriormente en Valencia), apuntando que este catedrático gaditano dirigió tres tesis, todas con la máxima calificación: "la de Gómez Marcano Encefalitis guanidinica, la de Pérez-Llorca Origen del cuerpo vítreo y la de Gil Olarte Hipernefromas". En el entorno de Luis Urtubey también estuvieron, entre otros, Marí Martínez y Álvarez de la Torre (Gómez Sánchez 2008, p. 42, 6364 y 89-90).

Una vez defendida la tesis doctoral, Antonio Gómez Marcano busca una revista adecuada para publicarla, y nada menos que consigue que se la publiquen en 1934 en Archivos de Neurobiología, revista fundada en 1919 por José Ortega y Gasset, Gonzalo Rodríguez Lafora

DOI del artículo:

http://dx.doi.org/10.25267/Cuad investig fondos arch UCA.2019.i1 .03 
y José M. Sacristán. La revista en 1934 es el órgano oficial de la Asociación Española de Neuropsiquiatras y de la Sociedad de Neurología y Psiquiatría de Madrid, y se editaba en el número 12 de la madrileña Plaza de Santa Ana (Ruiz Hermanos, editores). En octubre de 1934, Santiago Ramón y Cajal constaba como Presidente del Comité de Redacción de la revista, aunque como es sabido falleció en ese mismo año, después de haber sembrado e impulsado una formidable escuela de neurohistólogos y neurohistopatólogos, entre los que se podrían citar a Jorge Francisco Tello, Rafael Lorente de No o Fernando de Castro, entre otros, sin olvidarnos de su complicada e interesante relación con Pío del Río Hortega (1882-1945), discípulo y continuador de la obra de Nicolás Achúcarro Lund (1880-1918) (López-Piñero 2009, p. 541-553). Pío del Río Hortega prologó a Luis Urtubey, director de la tesis de Marcano, su excelente libro de Elementos de Histología. Creemos que la relación entre Urtubey y Río Hortega tuvo también algo que ver en la formación histoquímica de Antonio Gómez Marcano como tendremos ocasión de comentar.

Así pues, Marcano, tuvo un excelente director de tesis doctoral, Luis Urtubey; encontró el ambiente científico adecuado en Cádiz para llevar a cabo su investigación, que fue acogida para su publicación en una de las revistas más prestigiosas de la época. La tesis vio la luz pública en 1934, en el tomo XIV, número 3 de la citada revista, y se realizó de la misma una separata cuya portada reproducimos en la Imagen 2.

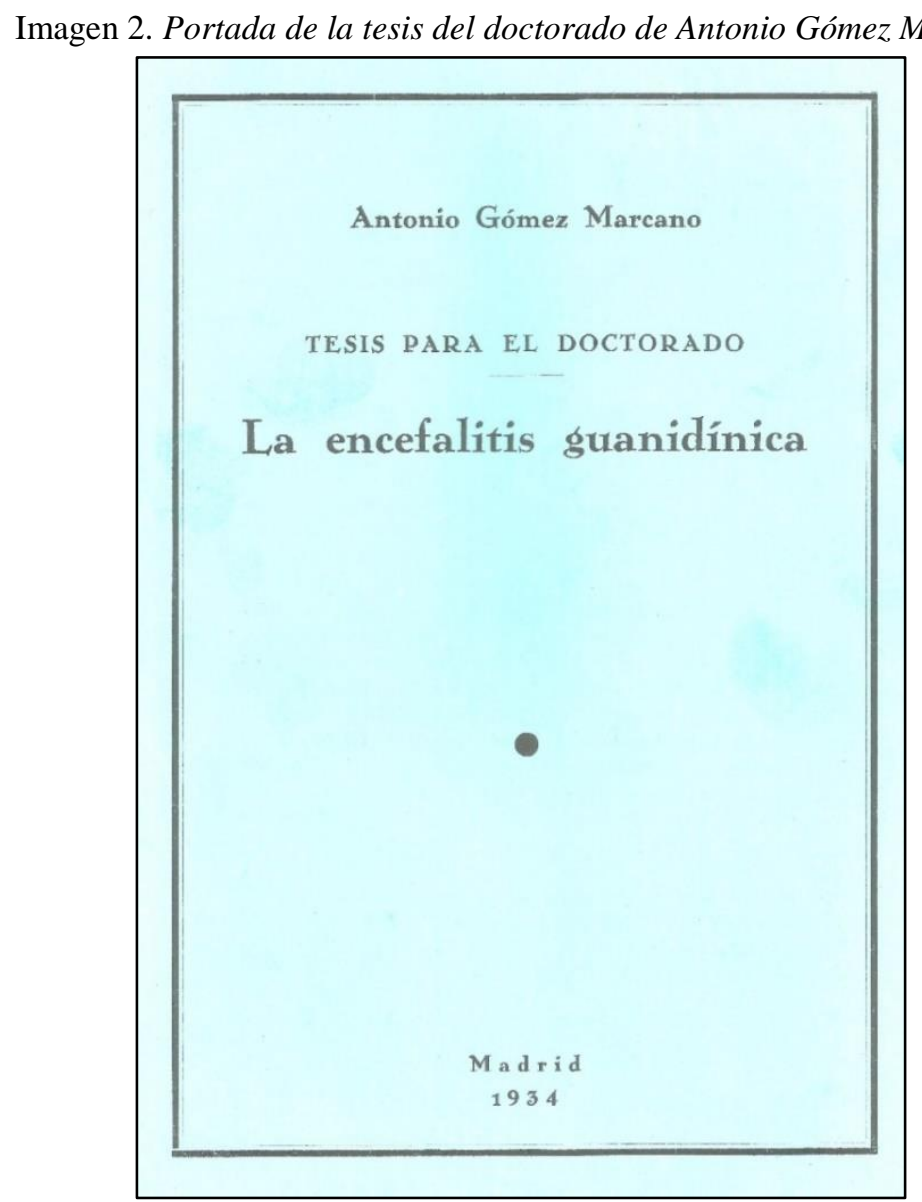

Fuente: Separata publicada por Archivos de Neurobiología (1934). Archivo de Francisco Herrera. 
En el comienzo de su tesis doctoral, Antonio Gómez Marcano, establece tres claves fundamentales para entender la génesis de su investigación: primero, el magisterio de Luis Urtubey en su servicio de Histopatología de la Facultad de Medicina de Cádiz; segundo, su estancia en Madrid para el "adiestramiento personal en las técnicas con el carbonato de plata de Río Hortega, adquirido en el propio Laboratorio de este ilustre histólogo español durante una brevísima asistencia al mismo"; y tercero, la lectura de un trabajo sobre patogenia hiperguanidinémica de la tetania, "que nos hizo venir a la imaginación la utilidad de hacer un parangón entre los hallazgos histológicos descritos en los animales paratireoprivos y en los intoxicados con guanidina”. Gómez Marcano establece un problema de investigación, para el cual deberá establecer objetivos y un diseño experimental, que incluya la selección de animales y los métodos adecuados de tinción para la observación microscópica, así como las dosis y las frecuencias con que se ha administrado la sustancia empleada. El objetivo de la investigación es estudiar histopatológicamente los centros nerviosos en animales intoxicados con guanidina, queda fuera de este trabajo específico realizar investigaciones análogas sobre los centros nerviosos de animales en tetania por ablación experimental de las paratiroides. Sobre los antecedentes de investigaciones que relacionan paratiroides, guanidina y tetania, se apoya en los trabajos previos de Patón, Findlay y Watson (1917), en las comunicaciones de Frank, Stern y Nothman (1921), los trabajos de Mac Callum, Bayer y Form (1924), Susman (1927), Traut y Macfate (1931). No olvida que fue Alois Alzheimer quien primero dedicó atención al problema histopatológico de la tetania y que en 1913 Moellgaard describió en la tetania lesiones de las astas anteriores de la médula cervical y lumbar, corroboradas por otros autores como Eppinger, Falta, Rudinger, Weiss y Zappert.

$\mathrm{Y}$ en lo que se refiere a los hallazgos histopatológicos en los animales intoxicados con guanidina, apunta Marcano que los primeros trabajos en este sentido son los de Rosenthal (1913), "que comprobó la existencia de una meningoencefalitis" en los animales intoxicados con la citada sustancia. Posteriormente, en 1921, fue Pollak quien se dedicó a realizar investigaciones sobre la guanidina como productora de alteraciones del sistema nervioso central, siendo este trabajo importante para Marcano en su investigación. Pollak se sirvió en sus experimentos de "gatos intoxicados con guanidina", aunque su trabajo incurre en un defecto metodológico ya que no indica las dosis; en cuanto a los colorantes del tejido nervioso utilizó el azul de toluidina, la tionina y los métodos de coloración glial de Alzheimer, Olivecrona y Jakob. Pollak fundamentalmente encontró “mayor acción nociva del tóxico” en el cerebro, aunque también encontró lesiones en el resto de los centros nerviosos. Marcano resume así la valoración que hace Pollak de los resultados de sus investigaciones en los gatos intoxicados con la guanidina: Factor alterativo (degeneraciones neuronales); factor exudativo (presencia de linfocitos y Plasmazellen, en meninges y en torno de los vasos; y factor proliferativo (gliosis y neuronofagia, por la glía).

Antonio Gómez Marcano sabe que el capítulo de material y métodos de su tesis debe ser preciso y meticuloso, de hecho debía ser consciente de que no podía incurrir en el error de Pollak que no indicó la dosis, como ya hemos señalado. Marcano utiliza como "material de experimentación el conejo común” y lo justifica "por la facilidad con que se impregna en

DOI del artículo:

http://dx.doi.org/10.25267/Cuad investig fondos arch UCA.2019.i1 .03 
este animal la microglía”. El tóxico que emplea es el clorhidrato de guanidina purísimo de Kahlbaum, en solución acuosa, inyectado subcutáneamente en el tejido celular del abdomen, a dosis progresivamente crecientes. Se efectuaron intoxicaciones agudas y crónicas, siendo éstas de diferente duración, según se expone en la Imagen 3. En lo que se refiere a la intoxicación guanidínica aguda contó con la colaboración del Dr. José Pérez Llorca (19021996) (Díaz-Rubio 2003, pp. 134-135), que como hemos indicado también realizó la tesis doctoral con el profesor Luis Urtubey. De esta colaboración apareció una publicación, firmada por Gómez Marcano y Pérez Llorca, en la revista Archivos de Medicina, Cirugía y Especialidades, en 1933, titulada "La encefalitis guanidínica aguda experimental”, que se cita en la bibliografía de la tesis doctoral que estudiamos.

\begin{tabular}{|c|c|c|c|c|c|c|}
\hline Conejo & $39 / 214$ & 0,050 & gr. & en & 1 & día. \\
\hline$»$ & $62 / 214$ & 0,250 & $»$ & $\gg$ & 2 & días. \\
\hline$»$ & $16 / 215$ & 0,350 & $»$ & $\gg$ & 3 & $»$ \\
\hline$\gg$ & $73 / 214$ & 0,350 & $\gg$ & $»$ & 3 & $»$ \\
\hline$»$ & $32 / 214$ & 0,475 & $\gg$ & $\gg$ & 9 & $»$ \\
\hline$»$ & $62 / 215$ & 1,150 & $»$ & $» 1$ & 14 & $»$ \\
\hline 1 & $43 / 214$ & 1,205 & $\gg$ & $» 1$ & 16 & $\gg$ \\
\hline » & $5 / 214$ & 1,250 & $»$ & $» 2$ & 25 & $»$ \\
\hline$»$ & $97 / 214$ & 1,720 & $»$ & $» 2$ & 20 & $»$ \\
\hline$»$ & $33 / 215$ & 1,985 & $»$ & $\gg 2$ & 22 & $»$ \\
\hline$»$ & $83 / 215$ & 2,820 & $»$ & 113 & 36 & $»$ \\
\hline$»$ & $4 / 215$ & 3,050 & $»$ & $\gg 3$ & 31 & $»$ \\
\hline
\end{tabular}

Fuente: Gómez Marcano, 1934

El material anatómico utilizado procedía, pues, de los conejos muertos mediante vapores de cloroformo, extraído rápidamente en todos los casos; "también practicamos el examen de centros nerviosos de conejos no inyectados con guanidina, bien en estado normal, bien después de hacerles heridas cerebrales experimentales, valiéndonos de un punzón. El objeto de estos exámenes fue tener un índice morfológico de la microglía normal y en actividad fagocitaria, respectivamente, que nos sirviera de base objetiva para comparar los demás resultados".

En cuanto a las técnicas de tinción también es minucioso Marcano en la exposición de las mismas, sin duda busca que cualquier investigador pueda reproducir su investigación, como es preceptivo: "Las técnicas que hemos puesto en práctica han sido variadas. Para el estudio de las neuronas nos hemos valido principalmente del óxido de plata amoniacal de Bielchowsky. Para la neuroglia común, del oro sublimado de Cajal y del carbonato argéntico lítico de Río Hortega. Para la microglía, el carbonato de plata de Río Hortega, y la variante, con el tungstato argéntico de Romero Martínez. Por último, hemos ensayado teñidos de la oligodendroglía con la técnica del cromato de plata de Golgi, modificada por Río Hortega, y

DOI del artículo:
http://dx.doi.org/10.25267/Cuad investig fondos arch UCA.2019.i1
.$\underline{03}$

Editorial ad UCA 
también con el método Golgi-Kopsth-Zimmermann, que se emplea comúnmente para el estudio de los pericitos".

También debemos reseñar que las fotomicrografías (palabra que emplea Gómez Marcano correctamente, no como en algunos trabajos actuales en que son denominadas microfotografías), fueron realizadas por el profesor auxiliar de la cátedra Rafael Calbo Cuadrado, sobre cuyo trágico final haremos referencia más adelante en este artículo. Un ejemplo del trabajo fotomicrográfico de Calbo Cuadrado se puede apreciar en la Imagen 4, en la que habiéndose empleado el método de Bielchowsky se aprecian “intensas lesiones de los elementos neurales, afectando éstos el aspecto de hinchazón y vacuolización citoplásmicas, que se presenta frecuentemente en las toxicosis aguda”. Todo lo cual revela que el clorhidrato de guanidina inyectado subcutáneamente ejerce una acción tóxica sobre los centros nerviosos, "manifestada muy precozmente, como pudimos comprobar en el conejo 16/215, inyectado con gramos 0,25 en dos días".

Imagen 4. Cerebro del conejo 16/215: Sustancia gris. Óxido de plata amoniacal. Neuronas alteradas,

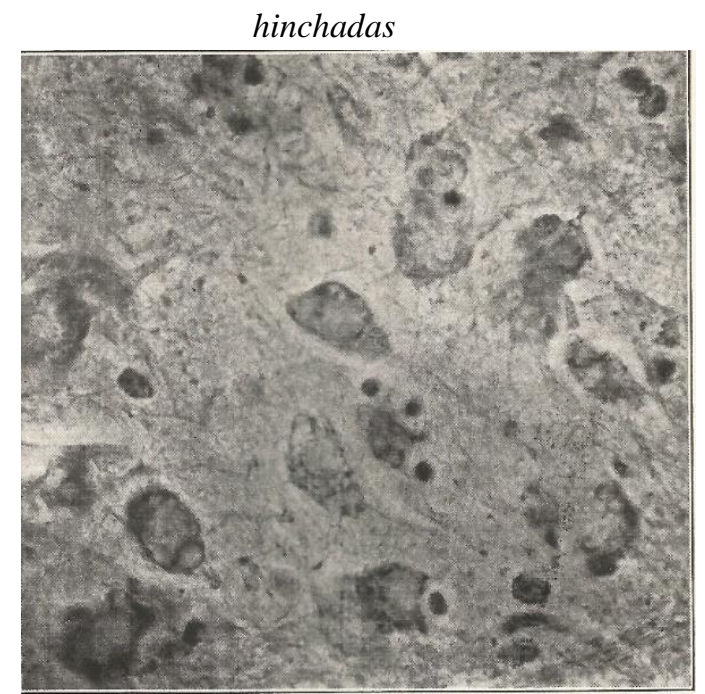

Fuente: Fotomicrografía R. Calbo Cuadrado (Gómez Marcano 1934).

En lo referido a animales con intoxicación guanidínica crónica, explica Marcano que han sido sometidos a la acción de una cantidad aproximada de tres gramos de guanidina (clorhidrato), en período también aproximado, "de veintitantos a treinta días". El doctorando sometió sus resultados a discusión, comparándolos con otros autores, después de lo cual llega a las siguientes conclusiones, que reproducimos íntegramente por su claridad expositiva:

"1 ${ }^{a}$. En los animales sometidos a intoxicación por el clorhidrato de guanidina hemos encontrado: alteraciones parenquimatosas por parte de las neuronas, ya de tipo de hinchazón (intoxicación aguda), como de tipo de retracción (intoxicación crónica); alteraciones parenquimatosas por parte de la neuroglia, que en uno y otro caso han consistido en degeneraciones y gliosis; alteraciones mesenquimatosas de tipo exclusivamente microglial"

DOI del artículo:
http://dx.doi.org/10.25267/Cuad investig fondos arch UCA.2019.i1
.03


$2^{a}$. Nos ha sido imposible hallar los fenómenos de infiltración meníngea y perivascular descritos por Pollak en el gato, en análogas condiciones.

$3^{a}$. Las lesiones observadas, tanto alterativas como reaccionales, se han presentado en el cerebro con mayor intensidad que en los demás centros nerviosos.

$4^{a}$. En ausencia de toda modificación meníngea, pero en presencia dentro del tejido nervioso de los factores necesarios para conceptuar una inflamación, estimamos que en los conejos la intoxicación guanidínica produce una típica 〈encefalomielitis〉’”.

La tesis doctoral de Gómez Marcano cuenta con 45 referencias bibliográficas, incluyendo la que él mismo firma con Pérez-Llorca. El 71\% de estas citas bibliográficas corresponden a trabajos publicados entre 1923 y 1933. Entre estas se incluyen 4 de Santiago Ramón y Cajal, dos de las mismas referidas al estudio de la neuroglia; 12 de Pío del Río Hortega, tanto de técnicas de tinción, como estudios específicos sobre la microglía, lesiones elementales de los centros nerviosos, neuroglia perivascular, etc., trabajos de don Pío publicados entre 1919 y 1928; asimismo, incluye a otros autores como Abaunza, Alberca, Belmonte Vento, Collado, Costero, Falta, Herrera Bollo, López Enríquez, Bratiano, Llombart y Romero Martínez. Y a autores como Aschoff, Bazgan, Bouin, Curschmann, Kramer, Levi, Mac Ciellan, GoodPasture, Moellgaard, Policard, Rosenthal, Rasdolsky, Revello y Spiegel.

Además del trabajo de Pollak, "Ueber experimentelle Encephalitis", publicado en 1921, que sirvió de base al estudio de Gómez Marcano, y del que concluye que este autor está vinculado "a la concepción rígida de la escuela morfologista y exigía, con arreglo al postulado de Nissl, la presencia de los tres factores para la conceptuación del proceso total como inflamatorio”. Conviene, según lo expuesto, hacer algunas precisiones más que ayuden a entender esta afirmación. Gómez Marcano se muestra ecléctico refiriéndose a la inflamación, distinguiendo en este debate a los "morfologistas" y a los "teleologistas". Señala que los "morfologistas" son muy exigentes en cuanto a las condiciones que estiman necesarias para calificar de inflamatorio un proceso en el sistema nervioso central. Señala a Nissl y Alzheimer, junto a otros autores, con quienes toma más incremento la idea de la necesidad de encontrarse reunidos los tres factores (alterativo, exudativo y proliferativo) "para que un proceso deba ser tenido como inflamatorio". Señala a Aschof como el autor que se opone a la "concepción sobradamente estrecha de los morfologistas, al supeditarla a un criterio estrictamente anatómico”. Para Aschof la inflamación es la capacidad que tienen todos los tejidos de reaccionar ante los "insultos", incluso los tejidos avasculares, sin que esta reacción haya de seguirse siempre de resultados beneficiosos. Considera Gómez Marcano que estas ideas son consecuencias de la concepción "teleológica" de la vida que impregna el pensamiento de este científico alemán. Marcano se sitúa en una "posición intermedia" que estima para la conceptuación de un proceso como inflamatorio la existencia de una reacción mesenquimatosa acompañando a una alteración cualquiera textural, preferentemente parenquimatosa, todo depende de "la índole de los componentes mesenquimatosos de los órganos afectos"; por eso piensa que en el Sistema Nervioso Central "la inflamación estará constituida por trastornos alterativos y reacción por parte del mesénquima de los centros, con presencia de factores exudativo y proliferativo reunidos o con la existencia de uno de

DOI del artículo:

http://dx.doi.org/10.25267/Cuad investig fondos arch UCA.2019.i1 Editorial ad UCA 
ellos aislado, que da carácter (exudativo o proliferativo) a la flegmasía". Toda esta problemática en torno a las investigaciones sobre la inflamación cuenta con un estudio del maestro de Gómez Marcano, Luis Urtubey, publicado en 1942, y también se ha realizado alguna síntesis muy interesante de este problema que intenta clarificar el debate planteado (Bullón Ramírez 1983, p. 13). Apunta lo siguiente el citado autor: "Consecuencia de la influencia de Virchow (...), fue la aparición de una viva polémica, que en realidad no ha terminado todavía, entre aquellos investigadores que consideran la inflamación con un sentido teleológico y proceso meramente defensivo, a la par que supuestamente beneficioso para el organismo y lo que los fieles al objetivo de toda ciencia, se esfuerzan por encontrar las causas que lo producen y también la de las consecuencias que durante su evolución puede producir en los tejidos y a cuyo acaecer, no se le puede dar el atributo de finalidad favorable para el organismo...".

Hasta aquí hemos expuesto las principales ideas y las conclusiones de la tesis doctoral de Gómez Marcano, procedemos seguidamente a exponer algunas circunstancias notables de su carrera profesional en la década de los treinta que creemos ayudarán a contextualizar un poco más su labor de investigación en Cádiz, así como los avatares padecidos como consecuencia de la Guerra Civil, que lo llevó al exilio en Orán y en Venezuela, país este último en el que realizó una importante labor en la lucha contra el paludismo

\section{CONSIDERACiONES FinAles: AÑos 30, GuERRA CiVIL Y EXILIO EN VENEZUELA.}

No cabe duda de que el Premio Nobel concedido a Santiago Ramón y Cajal, en 1906, supuso un revulsivo importante para establecer la Junta de Ampliación de Estudios, bajo la presidencia del propio Cajal. Una de las funciones de esta Junta era la preparación científica de los jóvenes, tanto dentro como fuera de España, y dotar becas por la llamada "Junta de Pensiones"; muchos de estos becados acudieron a formarse a Francia, Inglaterra, Alemania, USA, Austria, Suiza, Bélgica, Italia, etc., y fueron posteriormente figuras brillantes de la ciencia y de la medicina españolas, tan solo hay que recordar nombres como los de Gonzalo Rodríguez Lafora, Pío del Río-Hortega, Severo Ochoa, Roberto Novoa Santos, Rafael Lorente de No, Carlos Jiménez Díaz, Francisco Grande Covián y un largo etcétera que haría interminable este artículo (González Cajal 1989; Santamaría-García 2007, p. 1-21; HerreraRodríguez 2009b, p. 7). Además de médicos becados, también lo fueron químicos, matemáticos, filósofos o historiadores. No en vano los estudiosos denominan al periodo comprendido entre 1898 y 1936, por la dinamización científica o la producción literaria y artística llevada a cabo, Edad de Plata de la cultura española, precisamente algunos autores se han esforzado en sintetizar la producción en estos años en el terreno de las ciencias médicas (Albarracín Teulón 1994, pp. 495-544), y otros han analizado el proceso de institucionalización científica en España en el primer tercio del siglo XX (Ausejo 1993).

Pues bien, lo hemos comentado anteriormente, Antonio Gómez Marcano también recibió una de estas becas. El profesor Guerra ha señalado que en 1933 era Auxiliar de Anatomía

DOI del artículo:
http://dx.doi.org/10.25267/Cuad investig fondos arch UCA.2019.i1
.03

Editorial ad UCA 
Patológica en Cádiz con el profesor Luis Urtubey, su director de tesis, y que en 1934 fue pensionado dos años en Alemania por la Junta de Ampliación de Estudios, hecho que se puede corroborar en otras fuentes (Guerra 2003, p. 728; Junta de Ampliación de Estudios, 1935; González Cajal 1989, p. 106). En su expediente de profesor de la Facultad de Medicina, en el Archivo de la Universidad de Cádiz, se conserva un documento de la Subsecretaría de Universidades, dirigido al Rector de la Universidad de Sevilla, con fecha 29 de enero de 1935, en el que consta que se concede "a D. Antonio Gómez Marcano, por 8 meses y 28 días rehabilitación de pensión para continuar en Alemania sus estudios de Anatomía Patológica”. El propio Marcano, como hemos señalado, apunta también que tuvo una estancia breve en Madrid, en el laboratorio de Pío del Río Hortega, para aprender la técnica de tinción del carbonato de plata. No es sorprendente esta estancia suya en el citado laboratorio, ya que seguramente fue propiciada por su maestro Luis Urtubey, que suele ser reconocido como discípulo de Pío del Río Hortega (Oliva Aldamiz 1984, p. 184-186). El citado expediente personal de profesor de Antonio Gómez Marcano, en la Facultad de Medicina de Cádiz, corrobora que fue Ayudante de clases prácticas de Médica de $3^{\circ}$ curso (primero de octubre de 1930) y de Anatomía Patológica (primero de octubre de 1931), y según un documento firmado en noviembre de 1933 también fue Auxiliar de don Rafael Calbo Cuadrado, "toda vez que este se halla encargado del desempeño de la Cátedra vacante de Histología y Anatomía Patológica”. Hemos buscado en el Archivo Histórico de la Universidad de Sevilla, infructuosamente hasta el momento, un expediente de profesor de Antonio Gómez Marcano ya que la Facultad de Medicina de Cádiz dependía de esta Universidad, y en ocasiones hemos tenido la fortuna de encontrar expedientes de profesores de la Facultad gaditana tanto en esta ciudad como en Sevilla, ofreciendo el estudio de ambos documentos información complementaria. No ha sido este el caso, aunque como indicamos sí se ha podido consultar, como hemos visto, el existente en el Archivo de la Universidad de Cádiz. Si bien no ha aparecido en el Archivo sevillano el citado expediente, sí se ha localizado un documento del Rector de la Universidad de Sevilla dirigido al "Decano de la Facultad de Medicina de Cádiz”, fechado en Sevilla el día 22 de enero de 1936, en el que se transcribe un escrito del Ilmo. Sr. Subsecretario del Ministerio de Instrucción Pública y Bellas Artes, en el que señala que le ha sido concedida por la Junta de Ampliación de Estudios e Investigaciones Científicas, "la rehabilitación de pensión en el extranjero a D. Antonio Gómez Marcano, Profesor Auxiliar de la Facultad de Medicina de Cádiz de esa Universidad; esta Subsecretaría ha dispuesto que ese Rectorado oyendo a la Facultad de Medicina y con carácter de urgencia, informe sobre la conveniencia de dicha pensión y si la enseñanza queda debidamente atendida durante la ausencia de dicho Profesor Auxiliar”. En el apartado de Fuentes dejamos constancia de las referencias archivísticas para la consulta de este documento en el Archivo Histórico de la Universidad de Sevilla.

Todo lo apuntado hasta ahora nos lleva a situar a este joven y brillante médico gaditano, nacido en San Fernando, dentro de ese ambiente regenerador de la ciencia y de la cultura que en esos años se vivía en España, su tesis doctoral da testimonio de la calidad científica de su trabajo personal y de la del grupo de investigación en que estaba incardinado en la Facultad gaditana. Lastimosamente la Guerra Civil, como es sabido, generó muertes por doquier,

DOI del artículo:

http://dx.doi.org/10.25267/Cuad investig fondos arch UCA.2019.i1 .03 
sufrimientos, depuraciones y exilios, truncando el desarrollo científico y cultural que se venía desarrollando en el país en las primeras décadas del siglo XX.

Antonio Gómez Marcano, según ha apuntado Guerra, durante la Guerra Civil ejerció como médico de la Escuela de Aeronáutica Naval de Barcelona y luego como Jefe de Sanidad de la Base Naval de Cartagena. Sobre su destino en Barcelona se puede encontrar noticia en el Diario Oficial del Ministerio de Marina y Aire de 27 de octubre de 1936, que referenciamos en el apartado de Fuentes y Bibliografía. Al finalizar la Guerra se exilió en Orán y un año después se marchó a trabajar a Venezuela en la División de Malariología, Maracay, y a partir de 1944 fue coordinador de estudios en la Escuela de Malariología de Caracas (Guerra 2003, p. 728). Se ha señalado que Gómez Marcano recorrió, junto a Nieto Caicedo, "como médico de campo las distintas zonas para la erradicación de la malaria" y que fue profesor, junto con Gabaldón y Zozaya de la asignatura "Estudios del control de los vectores de las enfermedades metaxénicas" y que dictó la asignatura "Anatomía patológica de la malaria"; en 1950 "se encargó, además, de la coordinación de los cursos internacionales", jugando un papel importante en la docencia, en la investigación en los planes logísticos de la campaña con DDT iniciada en 1945 para la erradicación de la malaria (Martín Frechilla 2008, p. 534-535). Marquès Sureda y Martín Frechilla, en su excelente libro sobre la labor educativa de los exiliados españoles en Venezuela, señalan la importancia de estos médicos exiliados en la docencia de los cursos sobre la malaria, destacando a Antonio Gómez Marcano, Ángel Díaz Vázquez, Miguel Nieto Caicedo y Carlos Zozaya (Marquès Sureda y Martin Frechilla 2002, p. 173). En julio de 1956, Marcano participó en reuniones que tuvieron como objetivo discutir un proyecto de investigación clínica toxicológica, recomendamos para entender estas actuaciones el artículo "Sobre la necesidad de usar Dieldrín en Venezuela" (Berti, Gabaldon, Carrillo 1957, p. 502). Gómez Marcano, incluso, ejerció la dirección de la Escuela de Malariología y Saneamiento Ambiental durante el período 1964-1970 (Guevara de Sequeda y Marruffo García 2011, p. 115). La actuación, pues, de Gómez Marcano en la lucha contra la malaria en Venezuela fue importante y esto nos hace pensar que esta enfermedad también era endémica en algunos puntos de la provincia de Cádiz, y quizás nuestro médico sin las circunstancias trágicas de la Guerra Civil y su obligado exilio pudo haber colaborado con eficacia en la erradicación de la enfermedad en nuestro país, o quizás sus derroteros habrían seguido los caminos de la Histología, de la Histoquímica y de la Histopatología, como parecía apuntar su tesis sobre la encefalitis guanidínica, o habría seguido desarrollando su carrera médica en el seno de la Armada, pero claro está todo esto es hacer cábalas con lo que pudo haber sucedido si las cosas hubieran sido de otra manera, lo que sí está claro es que Antonio Gómez Marcano no podía quedarse en la España de Franco porque su vida habría estado en peligro. Gómez Marcano es un ejemplo, entre otros muchos más, de lo que perdió nuestro país en el terreno de la ciencia y de la medicina con esta guerra fratricida que consumió a España entre 1936 y 1939, sobre la que escribió un libro estremecedor Manuel Chaves Nogales: “A sangre y fuego. Héroes, bestias y mártires de España” (Herrera Rodríguez 2016).

Después de su jubilación Marcano volvió a España y residió en Cádiz hasta que falleció el 5 de julio de 1983 (Guerra 2003, p. 728). Unos días después, el 14 de julio, se celebró una

DOI del artículo:

http://dx.doi.org/10.25267/Cuad investig fondos arch UCA.2019.i1 .03 
misa "por el sufragio de su alma" en la parroquia de San Lorenzo de Cádiz, puede consultarse la esquela en Diario de Cádiz del día 14 de julio. Su hermano, Enrique Gómez Marcano, nacido en 1911, también en San Fernando, ejerció la medicina como "analista" y manifestó que no le gustaba hablar de la Guerra: “...por lo mal que se pasó, el hambre que soportó (fue la única vez que estuvo delgado, según decía) y porque en su familia había diferentes tendencias políticas" (Gil-Olarte Pérez et al. 2001, p. 162). Al volver a Cádiz, Antonio Gómez Marcano, debió traer consigo su biblioteca, sus libros o parte de ellos terminaron en esos asilos que solemos llamar librerías de lance o de viejo, en una de ellas tuve la fortuna de localizar su tesis doctoral y también un ejemplar del libro de Paul F. Rusell, "Malaria. Basic principles briefly stated", fechado y firmado por el propio Marcano en Maracay, Venezuela, en 1953 (Imagen 5).

\section{Imagen 5. Portada del libro de Paul F. Rusell sobre la Malaria (Oxford, 1952). Fechado y firmado por} Antonio Gómez Marcano en Maracay, Venezuela, 1953

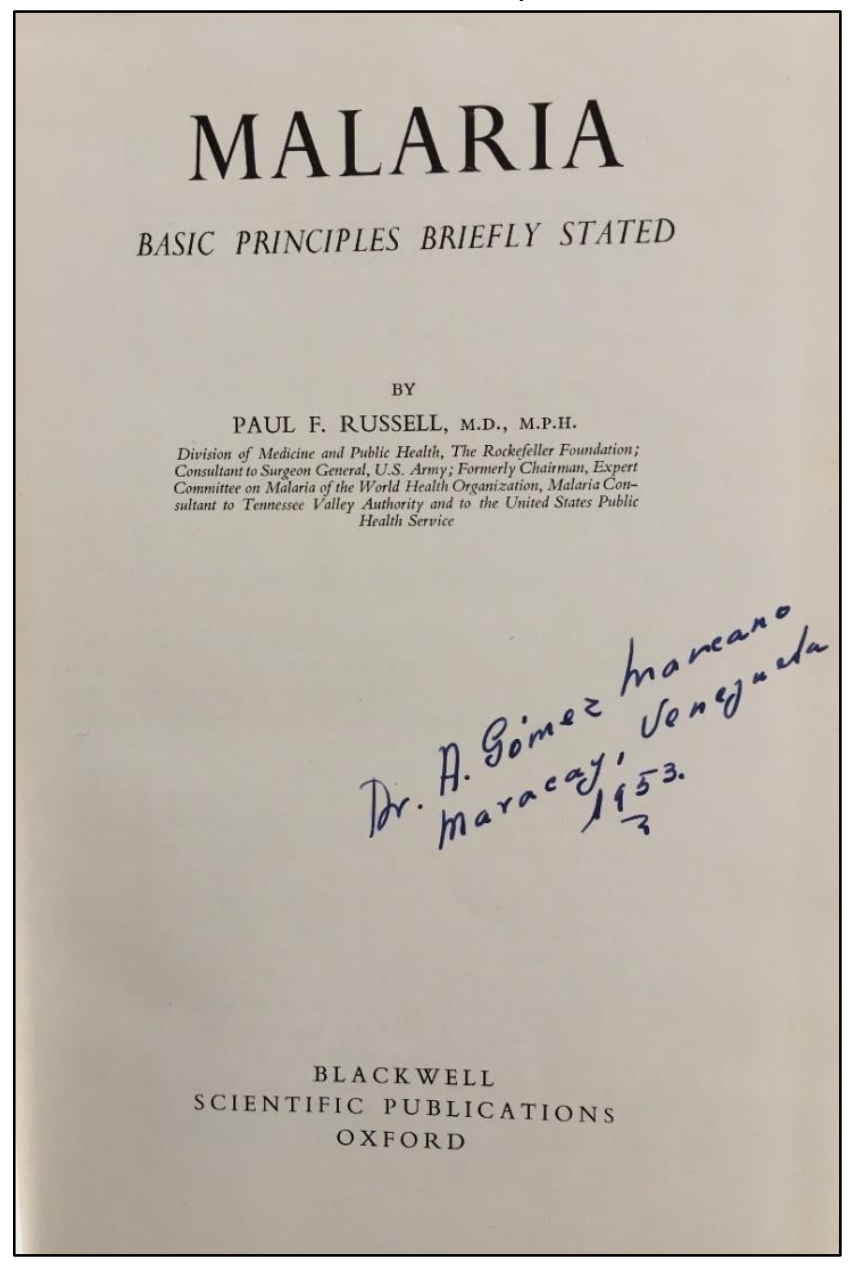

Fuente: Archivo de Francisco Herrera.

Un largo exilio el de Antonio Gómez Marcano, en Venezuela, como el de tantos otros médicos e intelectuales que se asentaron en este país, como por ejemplo el gran fisiólogo August Pi i Sunyer o el dermatólogo José Sánchez Covisa (Tinao Martín-Peña 2005, pp. 43-

DOI del artículo:
http://dx.doi.org/10.25267/Cuad investig fondos arch UCA.2019.i1
.$\underline{.03}$


54; Riera Palmero 1989, p. 251-261; Riera Palmero 2007, pp. 237-246; Gómez Sánchez 1989). Al leer la tesis doctoral de Marcano sobre la encefalitis guanidínica, defendida en 1933 y publicada en 1934, trabajo notable de investigación, hemos reflexionado no solo sobre la pérdida que supuso para la medicina gaditana y española su exilio en tierras venezolanas, como ya hemos indicado, sino también sobre el encarcelamiento y la pérdida de la cátedra de Histología de Valencia por parte de su maestro, el gaditano Luis Urtubey Rebollo, cuya vida ha sido biografiada por el profesor Gómez Sánchez, sin olvidarnos de los fusilamientos de su hermano Carlos Urtubey Rebollo, Jefe del Laboratorio Municipal de San Fernando, y del médico y diputado socialista Rafael Calbo Cuadrado (1906-1936), que ayudó en su tesis doctoral a Antonio Gómez Marcano realizando las fotomicrografías, una de las cuales reproducimos en este artículo como homenaje a su memoria (Imagen 4) (Gómez Sánchez 2008, p. 43; Domínguez-Pérez 2004: t. 1, p. 85; Álvarez-Rey, sin fecha; Álvarez Rey 2009, t. I, p. 493-499). De Rafael Calbo Cuadrado podemos apuntar que también defendió su tesis doctoral en la Facultad de Medicina de Madrid, en 1934, con un tema de investigación titulado "El disco germinativo y el área vascular de las aves", trabajo que aún no ha tenido la atención que se merece por parte de los investigadores. Su hermana, Margarita Calbo Cuadrado, fue depurada y repuesta en su cargo en 1938, jubilándose en 1973 (Pettenghi Lachambre 2005, p. 191). No podemos olvidar tampoco al médico Manuel de la Pinta Leal (1905-1936), alcalde de Cádiz y profesor de la Facultad de Medicina, que también fue fusilado, figura sobre la que van apareciendo estudios y del que se esperaba también una importante labor docente y política (Benítez-Aragón; Moreno-Tello y Núñez-Calvo 2012, p. 341-384; Ravina-Ripoll y Rodríguez-Moreno 2014, p. 159-175). Recientemente se ha publicado una biografía sobre el médico Daniel Ortega Martínez, que fue fusilado en Cádiz en agosto de 1941 (Almisas Albéndiz 2016). También debemos mencionar al médico jerezano Juan Planelles Ripoll (1900-1972), que se exilió en la Unión Soviética en 1939, desarrollando allí una importante labor científica y médica (Martínez-Hernández, 2014; Herrera-Rodríguez 2015, p. 178-180).

Esperamos que este artículo sobre el médico de San Fernando (Cádiz), Antonio Gómez Marcano, no sea el último, y que en el futuro podamos aportar nueva documentación para el mejor conocimiento de su figura, de momento quedan aquí estas páginas que tratan de ofrecer datos de un periodo de su vida, de 1919 a 1925, en que se formó como médico en la Facultad de Medicina de Cádiz, y de la década de los treinta, en que realizó en Madrid los cursos de doctorado y defendió su tesis doctoral con la que obtuvo la máxima calificación, además de sintetizar lo que han aportado otros investigadores sobre su exilio en Venezuela, país en el que tuvo una labor destacada como hemos podido apreciar en la lucha contra la malaria.

Agradecimientos: A María Clemencia, María José, Charo y Rocío, también por su ayuda en otros trabajos a Ana, Oliva, Antonia, Gertrudis y Berta, almas de ese lugar luminoso que es el Archivo y la Biblioteca de la Universidad de Cádiz. Y a Antonio Olivares, del Archivo Histórico de la Universidad Complutense, que tanto me ayudó hace más de dos décadas a buscar expedientes de doctorado, y concretamente el de Antonio Gómez Marcano.

DOI del artículo:
http://dx.doi.org/10.25267/Cuad investig fondos arch UCA.2019.i1
.$\underline{.03}$


Y también mi agradecimiento a las personas que me han atendido y ayudado en el Archivo Histórico de la Universidad de Sevilla.

\section{FUENTES DOCUMENTALES}

- Registro Civil de San Fernando (Cádiz). Partida de nacimiento de Antonio Gómez Marcano. Tomo 85, p. 358.

- Registro Civil de Cádiz. Partida de defunción de Antonio Gómez Marcano. Tomo 347, p. 480.

- Archivo de la Universidad de Cádiz (AUCA). Expediente personal del alumno Antonio Gómez Marcano. Medicina. C-211(3)-4 FM.

- Archivo de la Universidad de Cádiz (AUCA). Expediente personal del alumno Antonio Gómez Marcano. Escuela Profesional de Comercio de Cádiz. C-66-24 EC.

- Secretaría de la Facultad de Medicina/Archivo de la Universidad de Cádiz (AUCA). Expediente personal del profesor Antonio Gómez Marcano.

- Archivo Histórico de la Universidad Complutense de Madrid. Expediente académico de Antonio Gómez Marcano. Medicina. Caja 286.

- Archivo Histórico de la Universidad de Sevilla. Auxiliares de todas las Facultades. Asuntos generales. c. 1931-1946. Legajo 1996B-27.

- Archivo Francisco Herrera Rodríguez. Gómez Marcano, Antonio. Tesis para el doctorado. La encefalitis guanidínica. Archivos de Neurobiología. Tomo XIV (1934), 3, p. 461-492.

- Archivo Francisco Herrera Rodríguez. Gómez Marcano, Antonio. Tesis para el doctorado. La encefalitis guanidínica. Separata de Archivos de Neurobiología. Tomo XIV (1934), 3, p. 1-32.

- Archivo Francisco Herrera Rodríguez. Russell, Paul F. Malaria. Basic principles briefly stated. Blackwell Scientific Publications: Oxford, 1952. Libro con la firma del Dr. Antonio Gómez Marcano, fechado en Maracay, Venezuela, en 1953 (figura 5).

- Biblioteca José Celestino Mutis (Cádiz). Diario de Cádiz, jueves 14 de julio de 1983, p. 5 (Esquela de don Antonio Gómez Marcano).

- Diario Oficial del Ministerio de Marina: XXI (30 abril 1926), 96. [fecha de acceso 4 octubre 2018]. Sección de Sanidad. Resolución oposiciones al Cuerpo de Sanidad de la Armada. Disponible en internet: $\underline{\text { http://bibliotecavirtualdefensa.es/BVMDefensa/i18n/ }}$ catalogo_imagenes/grupo.cmd?path=116459

- Diario Oficial del Ministerio de Marina y Aire: XXXI (27 octubre 1936), 217. [fecha de acceso 4 octubre 2018]. Disponible en internet: $\underline{\text { http://bibliotecavirtualdefensa.es/ }}$ BVMDefensa/i18n/catalogo_imagenes/grupo.cmd?path $=124667$

- El Eco de Cartagena: LXVI (1 de mayo de 1926), 19.464. [fecha de acceso 4 octubre 2018]. Disponible en internet: http://hemeroteca.regmurcia.com/pdf.raw?query =id:0000168862\&lang=es

\section{REFERENCIAS BIBLIOGRÁFICAS}

DOI del artículo:
http://dx.doi.org/10.25267/Cuad investig fondos arch UCA.2019.i1
$\underline{.03}$

DOI del artículo: .03 
- Albarracín Teulón, Agustín. Las Ciencias Médicas. En: Jover Zamora, J.M. (dir.). Historia de España Menéndez Pidal. Tomo XXXIX, Volumen II, coord. LAín EnTRALGO, Pedro: La Edad de Plata de la cultura española (1898-1936). Madrid: Espasa Calpe, pp. 495544.

- Almisas AlbÉndiz, Manuel. Daniel Ortega Martínez, el médico comunista que revolucionó El Puerto. El Puerto de Santa María: Ediciones El Boletín, 2016. ISBN: 978-84-945532-0-2.

- Álvarez Rey, Leandro. Los diputados por Andalucía de la Segunda República 1931-1939. Diccionario biográfico [en línea]. Tomo I. Sevilla: Fundación de Centros Andaluces, p. 493499. [fecha de acceso 4 octubre 2018]. Disponible en internet: https://books.google.es/books?id=Fqyr32rqfccC\&pg=PA494\&lpg=PA494\&dq=rafael+calbo+

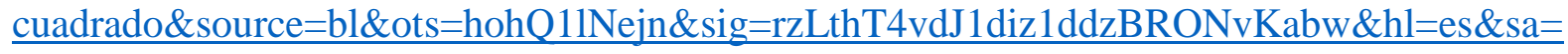
$\underline{X \& v e d=2 a h U K E w i P x Z j Q 7 v P d A h V O J B o K H Z b m B H M Q 6 A E w A n o E C A c Q A Q \# v=o n e p a g e ~}$ $\& q=$ rafael $\% 20$ calbo $\% 20$ cuadrado $\& \mathrm{f}=$ false

- Álvarez Rey, Leandro. Rafael Calbo Cuadrado. [en línea]. [Consulta: 4 octubre 2018]. Disponible en internet, sin fecha: http://www.todoslosnombres.org/content/biografias/rafaelcalbo-cuadrado

- AUSEJO, Elena. Por la ciencia y por la patria: la institucionalización científica en España en el primer tercio del siglo xx. La Asociación Española para el Progreso delas Ciencias. Zaragoza: Siglo XXI de España Editores, 1993. ISBN: 8432308048.

- Benítez Aragón, Rubén; Moreno Tello, Santiago y NúÑez Calvo, Jesús. Manuel de la pinta Leal, último alcalde de la Segunda República en Cádiz. En: Moreno Tello, Santiago (Ed.). La destrucción de la Democracia: Vida y muerte de los alcaldes del Frente Popular en la provincia de Cádiz. Sevilla: Junta de Andalucía y Diputación de Cádiz, Vol. 1, 2012, p. 341-384.

- Berti, Arturo luis; Gabaldón, Arnoldo; Carrillo, Salvador José. Sobre la necesidad de usar Dieldrín en Venezuela [en línea]. Boletín de la Oficina Sanitaria Panamericana. XLIII (1957), 6, p. 499-503. [Consulta: 4 octubre 2018]. Disponible en internet: http://iris.paho.org/xmlui/bitstream/handle/123456789/12284/v43n6p499.pdf?sequence=1

- Bullón Ramírez, Agustín. Historia de la inflamación. Madrid: Instituto de España. Real Academia Nacional de Medicina, 1983. ISBN: 84-7391-054-0

- Cannon, Dorothy F. Ramón y Cajal. Barcelona-México, D.F.: Ediciones Grijalbo, 1981. ISBN: 84-253-1305-8.

- DíAz Rubio, Manuel. Médicos españoles del siglo XX. $2^{\mathrm{a}}$ serie. Madrid: You \& US, 2003. ISBN: 84-96015-18-1.

- Domínguez PÉRez, Alicia: El verano que trajo un largo invierno. La represión políticosocial durante el primer franquismo en Cádiz (1936-1945). Tomo I. Cádiz: Quorum editores. ISBN: 84-88599-67-6.

- Gil-Olarte Pérez, Antonio; Márquez López, Amelia; Gil-Olarte Márquez, María Amelia; Gil-Olarte Márquez, María de los Ángeles; Gil-Olarte Márquez, Paloma. Figuras médicas de la Historia de Cádiz 1900-1950. Sevilla: Fundación Dr. Pascual, Diputación de Cádiz, Fundación El Monte. ISBN: 84-8455-033-8.

DOI del artículo:

http://dx.doi.org/10.25267/Cuad investig fondos arch UCA.2019.i1 .03 
- Gómez SÁnchez, José. Luis Urtubey: un maestro olvidado. Anales de la Real Academia de Medicina y Cirugía de Cádiz. (1980), p. 1-69.

- Gómez SÁnchez, José. Luis Urtubey: un maestro olvidado. Valencia: Consell Valencià de Cultura, 2008. ISBN: 978-84-482-4895-6.

- Gómez SÁnchez, José. Los histólogos españoles en América. En: Orozco AcuAviva, Antonio (dir.): Primeras Jornadas de Historia de la Medicina Hispanoamericana (Cádiz, 1983). Cádiz: Excma. Diputación Provincial de Cádiz, 1989, p. 263-276.

- González CAJAL, Jesús. Algunos datos sobre la psiquiatría que vivió Lafora en España hasta 1936 [en línea]. Revista de la Asociación Española de Neuropsiquiatría. IX (1989), 28, p. 101-113. [Consulta: 4 octubre 2018]. Disponible en internet: http://documentacion.aen.es/pdf/revista-aen/1989/revista-28/09-algunos-datos-sobre-la-

psiquiatria-que-vivio-lafora-en-espana-hasta-1936.pdf

- Guerra, Francisco. La medicina en el exilio republicano. Madrid: Universidad de Alcalá, 2003. ISBN: 84-8138-585-9.

- Guevara de Sequeda, Milady y Marruffo García, Marco. Una Escuela para la formación de expertos malariólogos. 75 años después...1936-2011. MeDULA. Revista de la Facultad de Medicina (Universidad de Los Andes). 20 (2011), 2, p. 102-116.

- Herrera RodríGuez, Francisco. La investigación científica en la Facultad de Medicina de Cádiz a través de las tesis doctorales producidas en la misma en el siglo XIX. Tesis doctoral. Microfichas. Cádiz: Servicio de Publicaciones de la Universidad de Cádiz, 1987a. ISBN 847786-950-2.

- Herrera Rodríguez, Francisco. Celestino García Fernández (1851-1908) y su tesis doctoral urológica (1870). En: Libro de Actas del "Congreso Internacional El Estrecho de Gibraltar". Ceuta: Universidad Nacional de Educación a Distancia, 1987b, p. 185-196.

- Herrera Rodríguez, Francisco. La tesis doctoral sobre la monomania y la pasión (1864) de Cayetano del Toro y Quartiellers (1842-1915). Anales de la Universidad de Cádiz. V-VI (1988-89), p. 73-84.

- HERRERA Rodríguez, Francisco. Estudio sobre el aborto quirúrgico a través de una tesis doctoral de 1872. Toko-Ginecología Práctica. L (1991), 7, p. 403-407.

- Herrera Rodríguez, Francisco. Un texto argentino sobre la gripe de 1918-1919 (tesis doctoral de José W. Tobias). Anales de la Real Academia de Medicina y Cirugía de Cádiz. XXVIII (1992), 1, pp. 283-299.

- Herrera Rodríguez, Francisco. La tesis doctoral sobre la lepra de Ángel Ferrer Cagigal (Cádiz, 1911). Llull. 18 (1995), p. 457-470.

- Herrera Rodríguez, Francisco. Luis Godoy y el beriberi [tesis doctoral,1896]. En: Herrera RodríGueZ, Francisco. Gavilla de médicos gaditanos. Cádiz: Quorum editores, 2000, p. 129-140.

- Herrera Rodríguez, Francisco. José Gómez-Plana y el estado sanitario del Distrito Médico de La Palma (Cádiz, 1929). En: PéRez-Bustamante, J.A. et al (dirs.). Actas del IX Congreso de la Sociedad Española de Historia de las Ciencias y de las Técnicas (Cádiz, 27-30 de septiembre de 2005). Cádiz: SEHCYT, 2006, tomo 1, p. 267-280.

DOI del artículo:
http://dx.doi.org/10.25267/Cuad investig fondos arch UCA.2019.i1
.$\underline{.03}$

DOI del artículo: .03 
- Herrera Rodríguez, Francisco. La tesis doctoral: ¿lombrosiana o utópica? En: Herrera RodríGUEZ, Francisco. La obra sanitaria de Leonardo Rodrigo Lavín (1867-1950). Cádiz: Servicio de Publicaciones de la Diputación de Cádiz, p. 31-40.

- Herrera Rodríguez, Francisco. El doctorado en la Facultad de Medicina de Cádiz en el siglo XIX. En: Estudios superiores en Cádiz desde 1748. Armada e Ilustración. Cádiz: Ministerio de Defensa y Universidad de Cádiz, 2009a, p. 203-217.

- Herrera Rodríguez, Francisco. Medicina y Sociedad en el reinado de Alfonso XIII. Anales de la Real Academia de Medicina y Cirugía de Cádiz. XXXII (2009b), 1 y 2, p. 5-14.

- Herrera Rodríguez, Francisco. Nuevos datos para la biografía de Luis Urtubey (18921962). En COBO, J.M. et al (editores). Actas X Congreso de la Sociedad Española de Historia de las Ciencias y de las Técnicas (Encuentro Europeo-Americano). Badajoz: SEHCYT, 2011, p. 449-459.

- Herrera Rodríguez, Francisco. Una lectura de la tesis doctoral de Gonzalo Rodríguez Lafora sobre la Enfermedad de Alzheimer (1914). Llull. 37 (2014), 80, p. 205-207.

- Herrera Rodríguez, Francisco. El médico rojo. Vida de Juan Planelles (reseña del libro de Juan Martínez Hernández). Llull. 38 (2015), 81, p. 178-180.

- Herrera Rodríguez, Francisco. A sangre y fuego. Héroes, bestias y mártires de España, de Manuel Chaves Nogales [en línea]. Temperamentvum (2016), 24. [Consulta: 4 octubre 2018]. Disponible en internet: http://www.index-f.com/temperamentum/tn24/t2404.php

- Herrera Rodríguez, Francisco y CABRERA AFONSO, Juan Rafael. La tesis doctoral sifilográfica de Rodolfo del Castillo y Quartiellers (1845-1917). Anales de la Real Academia de Medicina y Cirugía de Cádiz. XXII (1986), 1, p. 73-81.

- Herrera Rodríguez, Francisco; CABrera-Afonso, Juan Rafael; Márquez-EsPinós, Carlos. Pascual Hontañón Cabeza (1829-¿?) y su tesis doctoral sobre las escrófulas. En: Libro de Actas del "VIII Congreso Nacional de Historia de la Medicina (1986)". Murcia, 1988, vol. I, p. 313-327.

- Junta de Ampliación DE Estudios E Investigaciones Científicas. Memoria correspondiente a los cursos 1933 y 1934 [en línea]. Imprenta Góngora: Madrid, 1935. [Consulta: 4 octubre 2018]. Disponible en internet: http://cedros.residencia.csic.es/imagenes/ Portal/ArchivoJAE/memorias/014.pdf

- Laín Entralgo, Pedro. Escritos sobre Cajal. Edición de José Luis Puerta. Madrid: Editorial Triacastela, 2008. ISBN: 978-84-95840-35-6.

- LóPEz PIÑERo, José María. Cajal. Barcelona: Salvat Editores, S.A., 1985. ISBN: 84-3458209-6.

- LóPeZ PIÑERo, José María. Historia de la Medicina Española. Valencia: Ajuntament de Valencia, 2009. ISBN: 978-84-8484-303-0.

- MÁrquez Espinós, Carlos y Herrera RodríGuez, Francisco. Análisis de las tesis doctorales anestésicas gaditanas del Sexenio Revolucionario. Revista Española de Anestesiología y Reanimación. (1989), 36, p. 38-45.

- Marquès Sureda, Salomó y Martín Frechilla, Juan José. La labor educativa de los exiliados españoles en Venezuela [en línea]. Caracas: Fondo Editorial de Humanidad y

DOI del artículo:
http://dx.doi.org/10.25267/Cuad investig fondos arch UCA.2019.i1
$\underline{.03}$

DOI del artículo: .03 
Educación Universidad Central de Venezuela, 2002. [Consulta: 4 octubre 2018]. Disponible en internet: https://books.google.es/books?id=9BbTeJeQ51MC\&pg=PT176\&lpg=PT176

- Martín Frechilla, Juan José. El dispositivo venezolano de sanidad y la incorporación de los médicos exiliados de la Guerra Civil española. História, Ciéncias, Saúde-Manguinhos. 15 (2008), 2, p. 519-541.

- Martínez Hernández, Juan. El médico rojo. Vida de Juan Planelles. Madrid: "Ediciones 2010”, 2014. ISBN: 978-84-95058-10-2.

- Oliva Aldamiz, Horacio. Cajal y la anatomía patológica española, una historia compartida. Barcelona: Salvat Editores, 1984. ISBN: 84-345-2351-5.

- Orozco Acuaviva, Antonio. Bibliografía Médico-Científica gaditana. Ensayo biobibliográfico médico, científico y técnico de Cádiz y su provincia. Cádiz: Obra Cultural “Casino Gaditano", 1981. ISBN: 84-300-5165-1.

- Pettenghi Lachambre, José Aquiles. La escuela derrotada. Depuración y represión del Magisterio en la Provincia de Cádiz (1936-1945). Sevilla: Quorum editores, 2005. ISBN: 8488599-74-9.

- RAVina Ripoll, Rafael y RodríGuez Moreno, José Joaquín. La gestión municipal de Cádiz durante la Segunda República Española y la Guerra Civil Española. Las actuaciones del Alcalde republicano Manuel de la Pinta Leal y el alcalde franquista Juan de Dios Molina (1932-1940). Revista de Historia y Geografía. (2014), 31, p. 159-175.

- Riera Palmero, Juan. Fisiólogos catalanes en Hispanoamérica: La oba de Augusto Pi Suñer en Venezuela. En: Orozco Acuaviva, Antonio (dir.): Primeras Jornadas de Historia de la Medicina Hispanoamericana (Cádiz, 1983). Cádiz: Excma. Diputación Provincial de Cádiz, 1989, p. 251-261.

- Riera Palmero, Juan. Pío del Río Hortega en la Universidad de Valladolid. Arbor. Ciencia, Pensamiento y Cultura. (2005), 714, p. 181-197.

- Riera Palmero, Juan. La escuela catalana de fisiología y la obra de Augusto Pi y Sunyer. Revista Española de Investigaciones Quirúrgicas. X (2007), 4, p. 237-246.

- Riera Palmero, Juan y Del Río Hortega, Juan. Pío del Río Hortega y la institucionalización de la Ciencia en España. En: EsPAÑOL GonZÁLEZ, Luis y otros (coords.): Actas del VIII Congreso de la Sociedad Española de Historia de la Ciencias y de las Técnicas. Logroño: Universidad de La Rioja, 2004, vol. 1, p. 161-200.

- Rodríguez Sánchez, Juan Antonio. La supresión del Cuerpo de Médicos de Baños (19321936). En CAStellanos GUERRERo (Coord.). La medicina en el siglo XX. Estudios históricos sobre Medicina, Sociedad y Estado. Málaga: Sociedad Española de Historia de la Medicina, p. 269-280.

- Rodríguez SÁnchez, Juan Antonio; de Jorge García-Reyes, Javier; Crego Castaño, Manuela. Cien años haciendo ciencia: la cátedra de Hidrología Médica de la Facultad de Medicina de la Universidad Complutense (1912-2012). Catálogo de la exposición. Balnea. (2012), 7, p. 263-308.

- Santamaría García, Antonio. 100 años de investigaciones científicas. JAE-CSIC (19072007) [en línea]. CSIC, 2007, p. 1-21 [Consulta: 5 octubre 2018]. Disponible en internet: http://digital.csic.es/bitstream/10261/5290/1/100jae_csic.pdf

DOI del artículo:
http://dx.doi.org/10.25267/Cuad investig fondos arch UCA.2019.i1
.$\underline{.03}$


- Tinao Martín-Peña, José Francisco. Los médicos del exilio republicano en Venezuela. Historia Actual Online. (2005), 7, p. 43-54.

- Urtubey, Luis. La inflamación. Lecciones de Anatomía Patológica. Barcelona: Ediciones Científico-Médica, 1942. 